\title{
Summary of Workshop on Psychosocial Factors in Coronary Heart Disease: Measurement, Evaluation, and Intervention
}

\author{
J. Demirović* AND F. H. EPSTEIN $\dagger$ \\ * Department of Scientific Research and Development, University Clinical Centre, Pasterova 2, 11000 \\ Belgrade, Yugoslavia, and †Institute of Social and Preventive Medicine, University of Zürich, Switzerland
}

The European Society of Cardiology Working Group on Epidemiology and Prevention and the Yugoslav Society of Cardiology jointly sponsored this workshop which was held in Dubrovnik, Yugoslavia, on 23-25 April 1987.

A group of 23 experts (see Appendix) from various disciplines (epidemiologists, clinicians, psychologists, neuropsychiatrists, sociologists), together with a similar number of invited observers, met to assess the state of the art in the field of psychosocial factors and their role in coronary heart disease (CHD), to discuss conceptual and methodological problems in assessing these factors, to explore the prospects of using present results in the prevention of CHD, and to develop suggestions for future research.

Four main issues were addressed in the framework of panel discussions:

(1) Epidemiological and Clinical Approaches: Study Designs;

(2) Use of Questionnaires, Interviews, and Psychological Tests;

(3) 'Efferent Pathways': Biochemical and Physiological Measurements; and

(4) Intervention Studies.

A mini-session on 'Health Behaviour and Health Education' took place on the end of the workshop.

A selection of working papers prepared for the workshop are published in this issue of the European Heart Journal. The following is a short summary of the discussions and recommendations made, with suggestions for new research needs.

\section{Epidemiology of health behaviour}

Studies of the epidemiology of health behaviour in different population groups have been identified

Paper presented at the ESC Workshop on Psychosocial Factors in Coronary Heart Disease, Dubrovnik, Yugoslavia, 23-25 April 1987. as an area of high priority for future research, with the aims:

(1) to understand causes of risk behaviour, especially social causes;

(2) to identify high-risk groups;

(3) to explore potential links between social/ cultural factors and cardiovascular disease;

(4) to provide guidance for promoting changes in risk behaviour by means of 'conventional' health education or by other social/economic/ political approaches to change; and

(5) to examine the links between knowledge, attitudes, and behaviour as well as the reasons for non-compliance with health education, and with other kinds of intervention.

As most of the past and present epidemiological studies of psychosocial variables in CHD are concerned with middle-aged western European and American men, there is an ultimate need to assess these variables in different ethnic groups among both women and men and including younger age groups.

Design of studies linking psychosocial factors to disease risk

Cross-sectional epidemiological studies have limitations and are generally used to search for, but not to test, hypotheses. However, their broader use in the field of psychosocial factors should be kept in mind. Such studies may provide valuable data on the epidemiology of psychosocial factors in a wide sense, indicating which people behave in a healthy or unhealthy way, what are the group and individual differences, and how psychosocial factors vary with social structure, cultural patterns, level of economic development, and other influences.

In assessing the aetiological role of psychosocial factors in CHD, prospective study design is the 
design of choice, and these factors should be included in the baseline measurements of all new major prospective studies. CHD clinical end-points (angina, fatal and non-fatal myocardial infarction, sudden and non-sudden CHD death) need to be considered separately rather than as a group.

It is important to identify path models before using multivariate statistical methods to adjust for other known CHD risk factors: if a covariable is an intermediate step in a causal chain between the psychosocial factor of interest and the dependent variable, controlling for such a factor may diminish or eliminate the relationship between the psychosocial factor and CHD.

Repeated measurements of psychosocial variables over time would help to provide more accurate estimates of their impact on CHD morbidity and mortality, i.e. longitudinal effect of exposure. The recent development of statistical methods, which allows for multiple regression analysis using covariables that change over time, seems to hold much promise in analyzing longitudinal data.

Historical prospective study design may also be useful in assessing the relationship between the psychosocial factors and CHD, because a huge amount of data has already been collected.

In spite of a number of disadvantages, retrospective or case-control studies in assessing the relationship between the psychosocial factors and CHD (systematic and selection biases, effects attributable to the disease itself, recall bias, etc.) should not be neglected, particularly in evaluating secondary prevention measures related to psychosocial factors (such as counselling or drug therapy verified by medical records).

\section{Measurement}

A great deal of earlier research work on the relationship of psychosocial factors and CHD was concerned with the measurement of personality characteristics and type of behaviour. However, accumulating evidence indicates that both the individual and the social environment must be taken into consideration.

Much criticism has been addressed to the Type-A behaviour measures which are presently used in epidemiological studies. The general opinion was that different components of the Type-A behaviour construct should be considered separately in assessing their role in CHD. Potential for hostility and an inability or unwillingeness to express anger ('angerin') have been found as the 'toxic' components of the Type-A behaviour construct. As self-report measures do not accurately measure anger and potential for hostility, multi-method assessment of an individual Type-A behaviour has been recommended. It has been underlined that better standardization of Type-A behaviour pattern assessment is essential for future research work in this field.

Personality characteristics should be assessed in a dynamic model (coping style), especially in threatening/demanding situations rather then in the static models mostly used in the past.

Acute physiological reactivity to behavioural stimuli is an important variable to be measured as part of epidemiological studies. A standard definition of reactivity and instrumentation to measure reactivity are needed, as well as easily administered reactivity-eliciting procedures for use in such studies.

It has been well established that neuroendocrine reactions to behavioural stimuli involve catecholamines, corticosteroids, sex hormones, insulin, growth hormone, as well as a number of effects on carbohydrate, lipid and electrolyte metabolism. From the point of view of the feasibility of including these markers in the baseline measurements of the epidemiological studies, 24-h urine and plasma catecholamines and cortisol levels, lipid metabolism indicators, such as LDL and HDL, and coagulation indicators, such as fibrinogen, have been recommended, together with physiological measurements, such as 24-h blood pressure and ECG monitoring.

From the wide range of psychosocial variables relevant to $\mathrm{CHD}$, job characteristics and social support seem to be of prime importance, because they are affected by individual characteristics and are also closely bound with the social environment. In measuring and assessing job characteristics, it is necessary that modifications of the Karasek model are applied to non-industrial populations. The effects of work and family strain on women's cardiovascular health has been identified as an area of high priority for future research.

Social networks (i.e. the structure and quantity of social ties) and social support, which represents their function and quality, need to be measured in a more standardized manner. Future studies should aim to make a distinction between the main effect of social support (lack of which may be a stressor per se) and the buffer effect (lack of social support may only be harmful in the presence of a significant life stressor). 
Research is also needed in relating biochemical and physiological variables to the personality characteristics and coping style, to job characteristics (particularly to demand/decision latitude ratio), and to social support.

It has been underlined that, regardless of the content of the psychosocial variables assessed, standard tests and scales should be used which have documented inter- and intra-individual reliability and documented validation statistics.

\section{Intervention}

There is reasonably good evidence that psychosocial intervention can alter both classic and behavioural risk factors, and all new studies on multi-factorial risk intervention should include psychosocial-factor modification. Work on treating hypertension by stress management added to the belief that non-pharmacological intervention is possible. The examples taken from the experience gathered by the studies in which therapeutic modification of Type-A behaviour pattern was undertaken (including stress management, cognitive therapy, cognitive-behavioural therapy, and beta blockers) suggested that the recurrence of coronary events can be reduced.

Taking into account both the individual and social nature of cognition and emotions, the most promising approach for the future modification of health behaviour and prevention of CHD seems to be the utilization of the social support concept. Various techniques might be applied, such as family education, health education in patient groups which also provide stress management and social support, peer support and peer interaction, medical self-help groups, and others. There is some evidence that women are more likely then men to attend meetings which utilize social support techniques and that low-income groups have the highest dropout rate. Additional evidence from field trails are needed to justify extending the implementation of this concept to CHD prevention programmes at the population level.

Stress management at the working place has been identified as an area of great importance, and more practical and less costly methods to be applied in such settings are highly recommended.

Since health education plays an extremely important role in changing behaviour for health, success in stress management depends a great deal on understanding different models of health behaviour. It is crucial that educational principles are developed through the use of these models.

In conclusion, this workshop has emphasized again that a close collaboration between scientists from varied disciplines is essential to make progress in the very complex field of psychosocial factors and their effects on human health. General concern about the need for improved mutual communication and information led to the proposal for establishing a Working Party or Committee on Psychosocial Factors in collaboration with the European Society of Cardiology and the World Health Organization. One of the immediate tasks of such a Working Party would be to bring together researchers who already have data on psychosocial factors in order to try and bring their results under some common denominator.

It is hoped that these proposals will be put into action in the near future, so that psychosocial factors will cease to be 'second class' risk factors.

This workshop was also supported by the Council of Communities for Science of the SFR Yugoslavia, Administration for International Scientific, Educational, Cultural, and Technical Cooperation of SFR Yugoslavia, and The British Council.

\section{Appendix}

B. Badura (F.R.G.), S. Böthig (WHO), J. Demirović (Yugoslavia), E. Eaker (U.S.A.), F. Epstein (Switzerland), J. Gallacher (U.K.), J. Henry (U.S.A.), D. Johnston (U.K.), P. Kline (U.K.), M. Kornitzer (Belgium), M. Marmot (U.K.), R. Mulcahy (Ireland), S. Nedeljković (Yugoslavia), K. Orth-Gomer (Sweden), C. Patel (U.K.), K. Pyörälä (Finland), S. Rywik (Poland), N. Sartorius (WHO), I. Shkvatsabaya (U.S.S.R.), J. Siegrist (F.R.G.), T. Theorell (Sweden), G. Tibblin (Sweden), and M. Weiss (G.D.R.). 